\title{
THE EFFICIENCY OF DISINFECTANTS ON THE OOCYSTS OF EIMERIA TENELLA
}

EVA CHROUSTOVÁ, K. PINKA

Veterinary Research Institute, 62132 Brno

Received May 19, 1986

\begin{abstract}

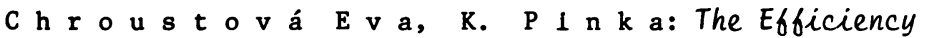
of Disinfectants on the Oocysts of Eimeria tenella. Acta vet. Brno, 56,1987: 141-149.

The effectiveness of selected disinfectants on the oocysts of Eimeria tenella was examined under laboratory and simulated field conditions. From the tested chemicals, which included ammonium hydroxide, formaldehyde, peracetic acid, glutaraldehyde and chloramine $B$ activated by ammonium hydroxide, the highest effectiveness was recorded in $5 \%$ ammonium hydroxide and 3 or $5 \%$ chloramine $B$ activated by 1 or $2 \%$ ammonium hydroxide. The $2 \%$ concentration of formaldehyde inhibited sporulation completely after 24 hour exposure, and was almost ineffective against sporulated oocysts.
\end{abstract}

Disinfection, coccidiosis, intensive poultry production, sporulated and non-sporulated oocysts.

The intensive poultry production in which thousands of birds are accumulated in a limited closed space, requires an efficient prevention of coccidiosis. Though the development of feed medication has provided conditions for rearing the poultry under intensive husbandry conditions, it is, however, often difficult to observe the recommended levels of anticocidial drugs in feed mixtures. In many cases, coccidiosis cannot be controled effectively even if anticoccidial drugs are used correctly.

Disinfection plays an important role in the complex of coccidiosis control. The purpose of disinfection is to destroy the exogenous forms of the parasite. Coccidial oocysts are very resistant structures. The oocyst wall consists of two chemically different layers and the combination of lipids and glycoproteins provides efficient protection of the germinal substance of the oocyst from the action of commonly used disinfectants (H 11 b r 1 c h 1975; E n $1 \mathrm{~g} k$ 1979; E r b e r 1980). Coccidial oocysts are resistant to all water-soluble disinfectants, even to the most agressive ones, such as $10 \%$ sodium-hydroxide or potassium hydroxide ( $\mathrm{H} \perp 1 \mathrm{~b}$ r $1 \mathrm{c} h$ 1975). Disinfectant effects on the oocysts can be expected only in lipid solving substances, such as phenol derivatives ( $\mathrm{H} i \mathrm{e} \mathrm{p}$ e et al. 1980). Heat destruction of oocysts, requiring temperatures over 70 to $80^{\circ} \mathrm{C}$, is not practical in large production units. 
In the present experiments, the action of several disinfectants on Eimeria oocysts was examined with the aim to recomend the most suitable ones for large poultry units infected with coccidia persistently.

\section{Materials and Methods}

Eimeria tenella cocysts were used for the evaluation of the disinfectants under laboratory and simulated field conditions. In the first step non-sporulated oocysts were treated and the effect on sporulation rate was used as a criterion. In this way it was possible to evaluate the effects of various concentrations and exposure periods quickly. The disinfectants showing the highest activity against non-sporulated oocysts selected for further experiments using sporulated oocysts and their activity was evaluated in biological tests on chicks.

The effect of disinfectants on non-sporulated oocysts

The following disinfectants were tested: 268 technical grade ammonium hydroxide, $36 \%$ peracetic acid (PERSTERIL), glutaraldehyde, chloramine B activated by ammonium hydroxide. The concentrations used are given in Tables 1 and 2 . The oocysts were either treated directly with the disinfectant solutions or exposed to vapours released from the solutions. As controls, untreated oocysts were used.

To examine the effect of the solutions, the centrifuged oocyst suspension was mixed with the solution tested and the mixture was left to stand for 1,4 or 24 hours at room temperature. After removal the disinfectant by washing, the non-sporulated oocysts were transferred into $2 \%$ potassium bichromate solution and allowed to sporulate at $27^{\circ} \mathrm{C}$ for 168 hours.

The effect of vapours was examined either in stoppered test tubes or in cylindrical glass cuvettes (diam. $10 \mathrm{~cm}$ ) with tight covers, containing 2 and $10 \mathrm{ml}$ of the solution tested, respectively.

In the first case the oocysts suspension was applied onto a bent strip of filter paper, which was placed into the test tube after drying off.

In the latter one an open Petri dish containing the oocyst suspension was placed into the cuvette (one was always taken to avoid the direct contact of the disinfectant solution with the oocysts)./

After, 1, 4 or 24 hours of exposure at room temperature the oocysts were allowed to sporulate as described above.

The effect of disinfectants on sporulated oocysts

The following disinfectants were tested: 268 technical grade ammonium hydroxide and $40 \%$ formaldehyde in liquid and vapour form, vapours of $36 \%$ peracetic acid, and chloramine $B$ activated by ammonium hydroxide. The concentrations tested are given in Table 3 and 4 . Following the exposure for $24 \mathrm{~h}$ at room temperature the oocysts were washed and used in biological tests on 5 - 14 days old chicks.

10000 or 25000 oocysts, suspended in $0.5 \mathrm{ml}$ water, were introduced into the crop of chicks via a metal tube. As controls, chocks infected with untreated oocyst cultures were used. The size of groups varied between 6 and 25 birds. To avoid spontaneous infection, the chicks were left in incubators and transferred in groups into wire cages immediately before being infected. The chicks were killed and necropsied one week p.i.

Lesion score ( $\mathrm{J} \circ \mathrm{h} \mathrm{n}$ s $\mathrm{n}$ and $\mathrm{R}$ i e d 1970) and death. rate due to coccidiosis were used as criteria for the calculation of the effectiveness tested. Further the oocyst count in caecal contents was evaluated. All data obtained were processed statistically using the Student's t-test. 
Table 1

The efficiency of disinfectants on the unsporulated Bimeria tenella cocysts

\begin{tabular}{|c|c|c|c|c|c|c|}
\hline \multirow[t]{2}{*}{ Substance } & \multirow{2}{*}{$\begin{array}{l}\text { Concen- } \\
\text { tration } \\
(x)\end{array}$} & \multirow{2}{*}{$\begin{array}{l}\text { Exo- } \\
\text { sure } \\
\text { (h) }\end{array}$} & \multicolumn{4}{|c|}{$\begin{array}{l}\text { Substance from } \\
\text { l1quid }\end{array}$} \\
\hline & & & $\begin{array}{l}\text { sporu- } \\
\text { lat1on } \\
(x)\end{array}$ & $\begin{array}{l}\text { effici- } \\
\text { ency } \\
(x)\end{array}$ & $\begin{array}{l}\text { sporu- } \\
\text { lation } \\
(x)\end{array}$ & $\begin{array}{l}\text { effic1- } \\
\text { ency } \\
\text { (z) }\end{array}$ \\
\hline \multirow[t]{4}{*}{$\begin{array}{l}\text { technical } \\
\text { grade } \\
\text { ammonium } \\
\text { hydroxide }\end{array}$} & $\begin{array}{l}0.1 \\
0.2 \\
0.5\end{array}$ & $\begin{array}{r}1 \\
4 \\
24 \\
1 \\
4 \\
24 \\
1 \\
. \quad 4 \\
24\end{array}$ & $\begin{array}{c}96.0 \\
66.0 \\
40.7 \\
57.7 \\
38.7 \\
14.3 \\
2.3 \\
0 \\
0\end{array}$ & $\begin{array}{c}0: \\
30.5 \\
57.1 \\
39.2 \\
59.3 \\
84.9 \\
97.5 \\
100.0 \\
100.0\end{array}$ & $\begin{array}{r}76.4 \\
81.0 \\
45.4 \\
84.4 \\
42.3 \\
7.0 \\
20.0 \\
5.0 \\
0\end{array}$ & $\begin{array}{r}19.9 \\
15.1 \\
52.4 \\
11.5 \\
55.7 \\
92.7 \\
79.0 \\
94.7 \\
100.0\end{array}$ \\
\hline & $\begin{array}{r}1 \\
2 \\
.5\end{array}$ & $\begin{array}{r}1 \\
2 \\
24 \\
1 \\
2 \\
24 \\
1 \\
4 \\
24\end{array}$ & $\begin{array}{l}0 \\
0 \\
0 \\
0 \\
0 \\
0 \\
0 \\
0 \\
0\end{array}$ & $\begin{array}{l}100.0 \\
100.0 \\
100.0 \\
100.0 \\
100.0 \\
100.0 \\
100.0 \\
100.0 \\
100.0\end{array}$ & $\begin{array}{l}6.7 \\
4.0 \\
0 \\
0.3 \\
0 \\
0 \\
0 \\
0 \\
0\end{array}$ & $\begin{array}{r}93.0 \\
95.8 \\
100.0 \\
99.7 \\
100.0 \\
100.0 \\
100.0 \\
100.0 \\
100.0\end{array}$ \\
\hline & 10 & $\begin{array}{r}1 \\
4 \\
24\end{array}$ & $\begin{array}{l}0 \\
0 \\
0\end{array}$ & $\begin{array}{l}100.0 \\
100.0 \\
100.0\end{array}$ & $\begin{array}{l}0 \\
0 \\
0\end{array}$ & $\begin{array}{l}100.0 \\
100.0 \\
100.0\end{array}$ \\
\hline & 26 & $\begin{array}{r}1 \\
4 \\
24\end{array}$ & $\begin{array}{l}0 \\
0 \\
0\end{array}$ & $\begin{array}{l}100.0 \\
100.0 \\
100.0\end{array}$ & $\begin{array}{l}0 \\
0 \\
0\end{array}$ & $\begin{array}{l}100.0 \\
100.0 \\
100.0\end{array}$ \\
\hline $\begin{array}{l}\text { control } \\
\text { potassium } \\
\text { dichromate }\end{array}$ & & & 94.9 & & 88.2 & \\
\hline \multirow[t]{4}{*}{$\begin{array}{l}\text { formalde- } \\
\text { hyde }\end{array}$} & 2 & $\begin{array}{r}1 \\
4 \\
24\end{array}$ & $\begin{array}{c}77.3 \\
45.7 \\
0\end{array}$ & $\begin{array}{r}18.5 \\
51.9 \\
100.0\end{array}$ & $\begin{array}{l}77.0 \\
64.2 \\
26.7\end{array}$ & $\begin{array}{r}0.4 \\
16.9 \\
65.5\end{array}$ \\
\hline & 5 & $\begin{array}{r}1 \\
4 \\
24\end{array}$ & $\begin{array}{l}73.0 \\
1.3 \\
0\end{array}$ & $\begin{array}{r}23.1 \\
98.6 \\
100.0\end{array}$ & $\begin{array}{l}79.2 \\
70.2 \\
20.0\end{array}$ & $\begin{array}{l}0 \\
9.1 \\
79.1\end{array}$ \\
\hline & 10 & $\begin{array}{r}1 \\
4 \\
24\end{array}$ & $\begin{array}{l}1.6 \\
0 \\
0\end{array}$ & $\begin{array}{r}98.3 \\
100.0 \\
100.0\end{array}$ & $\begin{array}{l}84.9 \\
63.1 \\
10.9\end{array}$ & $\begin{array}{c}0 \\
18.4 \\
85.9\end{array}$ \\
\hline & 40 & $\begin{array}{r}1 \\
4 \\
24\end{array}$ & $\begin{array}{c}12.0 \\
0 \\
0\end{array}$ & $\begin{array}{r}87.4 \\
100.0 \\
100.0\end{array}$ & $\begin{array}{r}39.1 \\
4.1 \\
0.1\end{array}$ & $\begin{array}{r}49.4 \\
94.7 \\
100.0\end{array}$ \\
\hline $\begin{array}{l}\text { control } \\
\text { potassium } \\
\text { dichromate }\end{array}$ & & & 94.9 & & 77.3 & \\
\hline
\end{tabular}


Table 2

The efficiency of disinfectants on the non-sporulated Eimeria tenella oocysts

\begin{tabular}{|c|c|c|c|c|c|c|}
\hline \multirow[t]{2}{*}{ Substance } & \multirow{2}{*}{$\begin{array}{l}\text { Concen- } \\
\text { tration } \\
(\%)\end{array}$} & \multirow{2}{*}{$\begin{array}{l}\text { Expo- } \\
\text { sure } \\
(\mathrm{h})\end{array}$} & \multicolumn{4}{|c|}{$\begin{array}{l}\text { Substance from } \\
\text { liquid }\end{array}$} \\
\hline & & & $\begin{array}{l}\text { sporu- } \\
\text { lation } \\
(\%)\end{array}$ & $\begin{array}{l}\text { effici- } \\
\text { ency } \\
(\%)\end{array}$ & $\begin{array}{l}\text { sporu- } \\
\text { lation } \\
(\%)\end{array}$ & $\begin{array}{l}\text { effici- } \\
\text { ency } \\
(\%)\end{array}$ \\
\hline \multirow[t]{6}{*}{$\begin{array}{l}\text { peracetic } \\
\text { acid }\end{array}$} & 2 & $\begin{array}{r}1 \\
4 \\
24\end{array}$ & $\begin{array}{l}91.3 \\
92.3 \\
92.7\end{array}$ & $\begin{array}{l}3.8 \\
2.7 \\
2.4\end{array}$ & $\begin{array}{l}- \\
-\end{array}$ & $\begin{array}{l}- \\
- \\
-\end{array}$ \\
\hline & 2.5 & $\begin{array}{r}1 \\
4 \\
24\end{array}$ & $\begin{array}{l}90.7 \\
87.3 \\
87.0\end{array}$ & $\begin{array}{l}4.4 \\
8.0 \\
8.3\end{array}$ & $\begin{array}{l}92.0 \\
84.6 \\
72.8\end{array}$ & $\begin{array}{c}0 \\
4.1 \\
17.5\end{array}$ \\
\hline & 5 & $\begin{array}{r}1 \\
4 \\
24\end{array}$ & $\begin{array}{l}93.3 \\
91.7 \\
81.0\end{array}$ & $\begin{array}{r}1.7 \\
3.4 \\
19.0\end{array}$ & $\begin{array}{l}- \\
-\end{array}$ & $\begin{array}{l}- \\
-\end{array}$ \\
\hline & 10 & $\begin{array}{r}1 \\
4 \\
24\end{array}$ & $\begin{array}{l}90.7 \\
86.7 \\
68.0\end{array}$ & $\begin{array}{r}4.5 \\
8.7 \\
28.4\end{array}$ & $\begin{array}{l}87.9 \\
87.7 \\
22.0\end{array}$ & $\begin{array}{r}0.3 \\
0.6 \\
75.1\end{array}$ \\
\hline & 20 & $\begin{array}{r}1 \\
4 \\
24\end{array}$ & $\begin{array}{r}83.3 \\
81.7 \\
3.3\end{array}$ & $\begin{array}{l}12.2 \\
13.9 \\
96.5\end{array}$ & $\begin{array}{l}89.5 \\
73.8 \\
22.0\end{array}$ & $\begin{array}{c}0 \\
16.3 \\
75.1\end{array}$ \\
\hline & 36 & $\begin{array}{r}1 \\
24 \\
\end{array}$ & $\begin{array}{r}79.0 \\
56.0 \\
\end{array}$ & $\begin{array}{r}16.7 \\
41.8 \\
\end{array}$ & $\begin{array}{r}88.9 \\
68.2 \\
0.8 \\
\end{array}$ & $\begin{array}{c}0 \\
22.7 \\
99: 1\end{array}$ \\
\hline \multicolumn{3}{|l|}{$\begin{array}{l}\text { concrol } \\
\text { potassium } \\
\text { dichromate }\end{array}$} & 94.9 & & 88.2 & \\
\hline \multirow[t]{3}{*}{$\begin{array}{l}\text { glutaral- } \\
\text { dehyde }\end{array}$} & 2 & $\begin{array}{r}1 \\
4 \\
24\end{array}$ & $\begin{array}{l}96.3 \\
92.7 \\
93.7\end{array}$ & $\begin{array}{l}0 \\
2.4 \\
1.8\end{array}$ & $\begin{array}{l}- \\
-\end{array}$ & $\begin{array}{l}- \\
-\end{array}$ \\
\hline & 5 & $\begin{array}{r}1 \\
4 \\
24\end{array}$ & $\begin{array}{l}92.3 \\
92.9 \\
93.7\end{array}$ & $\begin{array}{l}2.7 \\
2.0 \\
1.3\end{array}$ & $\begin{array}{l}- \\
-\end{array}$ & $\begin{array}{l}- \\
-\end{array}$ \\
\hline & 10 & $\begin{array}{r}1 \\
4 \\
24\end{array}$ & $\begin{array}{l}92.7 \\
94.9 \\
92.9\end{array}$ & $\begin{array}{l}2.4 \\
0 \\
2.1\end{array}$ & $\begin{array}{l}- \\
-\end{array}$ & $\begin{array}{l}- \\
-\end{array}$ \\
\hline $\begin{array}{l}\text { control } \\
\text { potassium } \\
\text { dichromate }\end{array}$ & & & 94.9 & & - & \\
\hline $\begin{array}{l}\text { chlorami- } \\
\text { ne } B+ \\
\text { ammonium } \\
\text { hydroxide }\end{array}$ & $\begin{array}{l}5+0,5 \\
5+1.0+ \\
5+2.0 \\
3+2.0 \\
3+2.0\end{array}$ & $\begin{array}{l}24 \\
24 \\
24 \\
24 \\
24\end{array}$ & $\begin{array}{l}0 \\
5.0 \\
0 \\
0 \\
5.0\end{array}$ & $\begin{array}{r}100.0 \\
94.3 \\
100.0 \\
100.0 \\
94.3\end{array}$ & $\begin{array}{c}0 \\
- \\
- \\
0 \\
-\end{array}$ & $\begin{array}{c}100.0 \\
- \\
\overline{0} .0 \\
-\end{array}$ \\
\hline $\begin{array}{l}\text { control } \\
\text { potassium } \\
\text { dichromate }\end{array}$ & & & 88.0 & & - & \\
\hline
\end{tabular}

+ the oocysts placed during the disinfectant action in a stable 
Experiments in poultry houses

In empty poultry houses the following disinfectants were tested: 28 and $5 \%$ solution of ammonium hydroxide, and 3 and $5 \%$ solution of chloramine $B$ activated by $0.5,1.0$ or $2 \%$ ammonium hydroxide. Petri dishes or enamelled metal dish containing oocyst suspension were placed on the floor and the disinfectant was poured over the floor (including the dishes) at the rate of 0.51 per $1 \mathrm{~m}^{2}$. After $24 \mathrm{~h}$ the oocysts were collected, washed and used for experimental infection on chicks. The chicks were killed and necropsied one week p.i. as described above.

\section{Results}

The effectiveness of the disinfectants under laboratory conditions

As can be seen in Table 1 and 2, ammonium hydroxide proved to be the most effective disinfectant, its 0.5 to $1.0 \%$ solution or vapours killing 93 to 978 of non-sporulated oocysts within 1 hour of exposure. Effectiveness of various concentrations and exposure periods of formaldehyde varied between 18.5 to $100 \%$, while the peracetic acid proved to be effective only in 10 to $20 \%$ concentration, requiring 24 hours of exposure. The effectiveness of glutaraldehyde was very low.

Both $2 \%$ solution and vapours of ammonium hydroxide were highly effective (100 号) against sporulated oocysts (Tables $\widehat{3}$ änd 4$)$. The effectiveness of the combination of ammonium hydroxide ( 0.5 or $\left.2 \frac{\circ}{\circ}\right)$ with chloramine B ( 3 or $5 \frac{8}{8}$ ) varied between 92.3 to $100 \%$. Formaldehyde, a disinfectant used commonly in the poultry industry, proved to be almost ( $17.5 \%$ for the $5 \%$ solution) or completely ( 28 solution) inactive.

The effectiveness of the disinfectants under simulated field conditions

The experiments performed in the poultry house of the institute confirmed the very high effectiveness (100\%) of both $5 \frac{\circ}{\circ}$ ammonium hydroxide and 3 or $5 \frac{\circ}{\circ}$ chloramine $\mathrm{B}$ activated by 1 or $2 \frac{\circ}{0}$ ammonium hydroxide (Table 5 ). No intestinal lesions caused by coccidia were found in the chicks infected with treated oocysts and also the search for oocysts in the caecal contents brought negative results. The effectiveness of chloramine $B$ activated by $0.5 \frac{0}{0}$ ammonium hydroxide was somewhat lower.

\section{Discussion}

The protection of day-old chicks against coccidiosis is, under current conditions, provided only through a mechanical cleaning of the premises and the equipment by warm water applied pressure of approximately $12 \mathrm{PMa}$. The disinfectants used commonly, between the individual crops are effective above all against bacteria and viruses, but not against the oocysts of coccidia. This applies especially to $2.5 \%$ formaldehyde in liquid or vapour form, which is generally regarded as a universally effective disinfectant. The results of our experiments showed that formaldehyde might - under certain conditions prevent the sporulation of $E$. tenella oocysts. However, being inactive against sporulated oocysts, which are predominant in infected poultry houses, it would not protect chicks against coccidiosis. In the infective stage, sporulated oocysts are much more resistant against adverse environmental factors than the non-sporulated ones.

$\mathrm{S} \mathrm{c} \mathrm{h} \mathrm{n}$ e i d e r et al. (1973) reported that the 5\% and 10\% formaldehyde solutions inhibited sporulation of $2-40 \%$ and $90 \%$ of $E$. tenella oocysts, respectively. On the other hand, E l-M o u k a d (1976) reported a very 


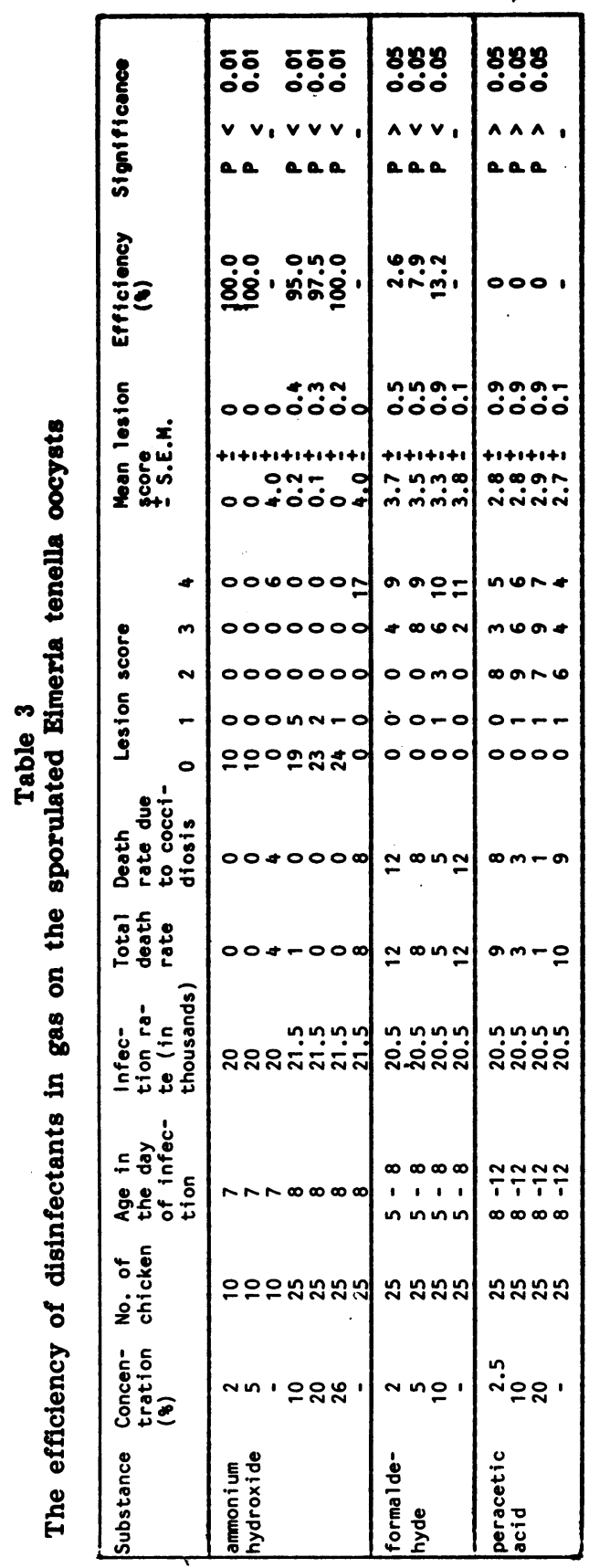




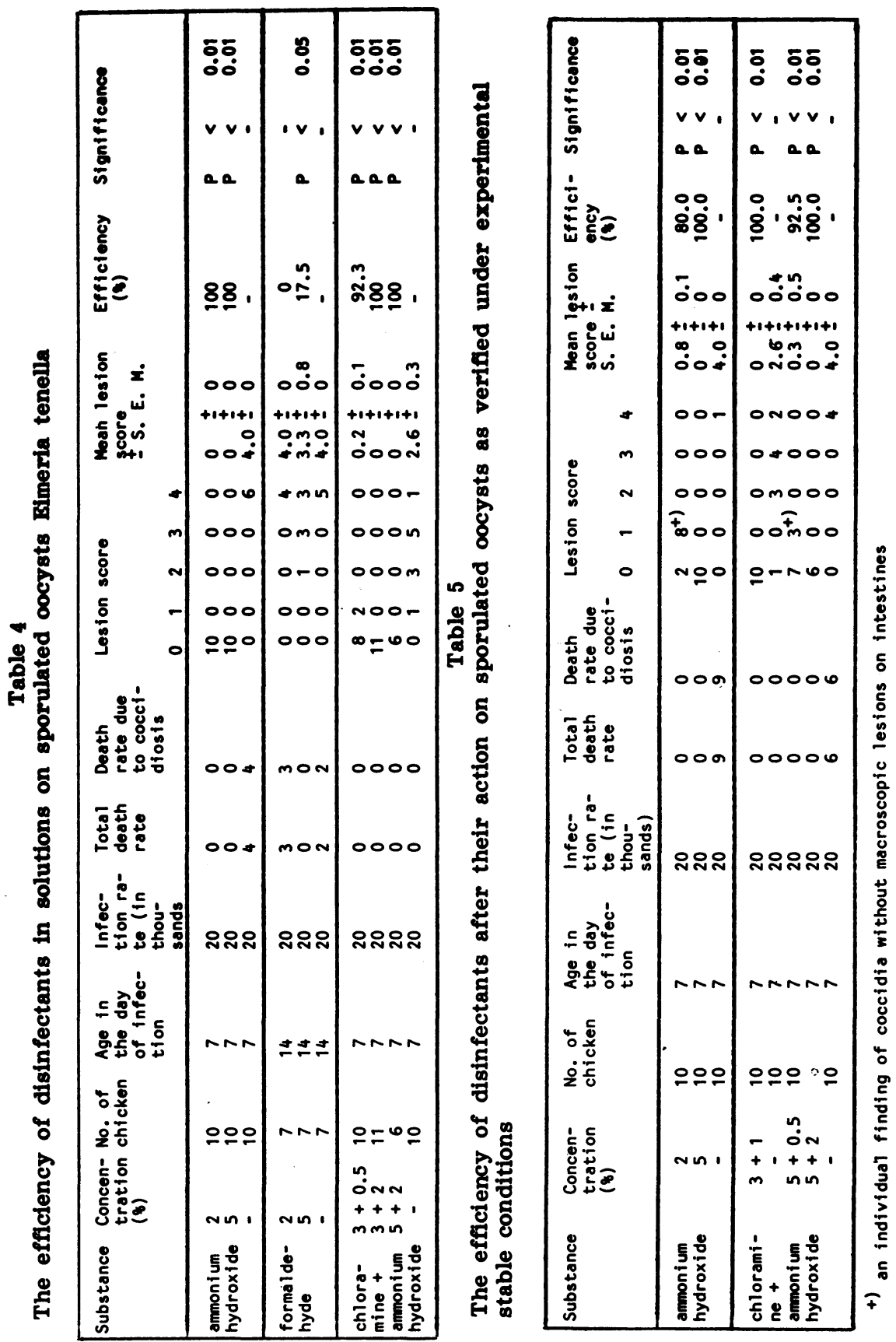


low effectiveness of formaldehyde. In his experiments, 108 concentration and exposure for $1 \mathrm{~h}$ was necessary to inhibit sporulation of $35-39 \&$ of $E$. necatrix and E.acervulina oocysts.

According to $\mathrm{E} \mathbf{n} \mathbf{i} \mathbf{g} \mathbf{k}$ (1979) oocysts of rabbit coccidia sporulated when kept in 58 water solution of formaldehyde at room temperature.

The effect of peracetic acid (PERSTERIL) on non-sporulated oocysts was very weak, as only oocysts exposed to 10 - 208 concentration for 24 hours were affected. Therefore this disinfectant was excluded from the experiments with sporulated oocysts. For the same reason the experiments with glutaraldehyde were discontinued.

Ammonium hydroxide, which proved to be highly effective in our experiments, was recommended for use in 78 concentration by $\mathrm{H} \circ \mathrm{j} \circ \mathrm{v}$ e c and $R \circ \mathrm{s}$ o c h a (1974). H i l b r i c h (1975), who investigated the effect of ammonium hydroxide on non-sporulated and sporulated $E$.tenella oocysts under exact experimental conditions, reported that both forms were inactivated completely by 2 a 48 solution within 2 - 30 minutes.

For the field use we recommend chloramine $B$ combined with ammonium hydroxide as an activator. This combination is effective against non-sporogenous and sporogenous bacteria, mycobacteria and some viruses ( $\mathrm{P}$ a v l a s 1967; $\mathrm{H} \circ \mathrm{j} \circ \mathrm{v} \mathrm{e}$ and $\mathrm{R} \circ \mathrm{s} \circ \mathrm{c} \mathrm{h}$ a 1974; $\mathrm{P} \check{\mathrm{r}} \mathrm{i} \mathrm{v} \circ \mathrm{r}$ a 1980) and the results of our experiments should be mixed 30 minutes before use and the solution should have a temperature of $15-20^{\circ} \mathrm{C}$.

For our experiments, we selected disinfectants used in veterinary medicine and especially in the poultry industry currently. Although only $E$. tenella oocysts were used, we believe that the results apply to the oocysts of the remaining poultry coccidia, too.

We do not assume that disinfection against coccidia should be performed in all poultry flocks routinely. However, there are poultry farms, in which persistent coccidiosis has a considerable economic impact. Physical measures (heat or drying) being not practicable in large poultry units, chemical disinfection is the only way how to destroy the exogenous coccidia forms. We suggest that, in persistently infected flocks, this preventive measure is of the same importance, as feed medication with anticoccidial drugs.

\section{Učinnost desinfekčních prostředků na oocysty Eimeria tenella}

V laboratorních a poloprovozních podmínkách byla ověrována účinnost vybraných desinfekčních prostředků na oocysty Eimeria tenella. Ze zkoušených látek - hydroxid amonný, formaldehyd, kyselina peroctová, glutaraldehyd, chloramin B aktivovaný 1 resp. $2 \%$ hydroxidem amonným - vykázal nejvyšši úcinnost hydroxid amonný $v 5 \%$ koncentraci a 3 resp. 58 chloramin $B$ aktivovaný 1 resp. $2 \%$ hydroxidem amonným. Formaldehyd zabránil sice ve $2 \%$ koncentraci po 24 hodinové expozici zcela sporulaci oocyst, avšak na vysporulované oocysty byl neúčinný.

\section{Воздействие дезинфицирующих средств на ооцысты Eimeria tenella}

В лабораторных и полузаводских условиях проверяли воздействие дезинфицирующих средств на ооцисты Eimeria tenella. Из испытываемых веществ - гидроокись аммония, формальдегид, перуксусная кислота, глютаральдегид, хлорамин В, активированний 1 или 28 гидроокиси аммония - самой большой еффективностью отличалась гидроокись аммония с концентрацией 58, а также 3 или 
58 хлорамина В, активированного 1 или 28 гидроокици аммония. Формальдегид в концентрации 28 хотя и в течени сусточного полноцтью препятствовал споруляции ооцистов, однако он не отличался еффективностью по отношению к сушествующим уже ооцистам.

\section{Acknowledgement}

The skillful technical assistance of Mrs. I. Kř́žková is highly appreciated.

\section{References}

EL-MOUKDAD, A.R.: Uber die Wirkung verschiedener Desinfektionsmittel auf präparasitäre Entwicklungstadien. Wien. tierärzt1. Mschr., 63, 1976:399405.

ENIGK, K.: Resistenz der Dauerformen von Endoparasiten der Haustiere. Ber1. Münch. tierärzt1. Wschr., 92, 1979: 491-497.

ERBER, M.: Wiederstandsfähigkeit von Kokzidienoozysten und-sporozysten sowie von Ascarideneiren gegen handelsübliche Desinfektionsmittel. Prakt. Tierarzt., $61,1980: 334-339$.

HIEPE, Th. - BUCHWALDER, R. - RIBBECK, R.: Lehrbuch der Parasitologie. 1. Auf1. Jena VEB Gustav Fischer Verlag 1981. Band 1.

HILBRICH, P.: Desinfektionsversuche an Eimeria-tenella-0ocysten. Ber1. Münch. tierärztl. Wschr., $88,1975: 144-148$.

HOJOVEC, J. ROSOCHA, J.: Hygiena hospodářských zvířat. Hygiena prostředí a jeho asanace. SPN Praha, 1974: 224 p.

JOHNSON, J. - REID, W.M.: Anticoccidial Drugs: Lesion scoring techniques in battery and floor-pen-experiments with chickens. Expl. Parasit., 28, 1970: 30-36.

PAVLAS, M.: Desinfekční účinnost chloraminu B a kyseliny peroctové na Mycobacterium bovis v prostředi čistém a znečištěném. Čs. Epidem. Mikrobiol. Imunol., 16, 1976: 282-286.

PŘIVORA, M.: Dezinfekce, dezinsekce, deratizace. Avicenum Praha, 1980: 220 p. SCHNEIDER, D. - AYENI, A.0. - DURR, U.: Zur Resistenz von Kokzidienoozysten gegen Chemikalien. Sammelreferat. Dte. tierärztl. Wschr., 80, 1973: 556$562,576-580$. 